EPJ Web of Conferences 12, 03004 (2011)

DOI: $10.1051 /$ epjconf/20111203004

(C) Owned by the authors, published by EDP Sciences, 2011

\title{
Qualification of a truly distributed fiber optic technique for strain and temperature measurements in concrete structures
}

\author{
J.M. Henault1, a , J. Salin ${ }^{1}$, G. Moreau ${ }^{1}$, S. Delepine-Lesoille ${ }^{2}$, J. Bertand², \\ F. Taillade ${ }^{3}$, M. Quiertant ${ }^{3}$ and K. Benzarti ${ }^{3}$ \\ ${ }^{1}$ EDF R\&D, 78400 Chatou, France \\ ${ }^{2}$ ANDRA, 92298 Chatenay-Malabry, France \\ ${ }^{3}$ Université Paris-Est, IFSTTAR, 75015 Paris, France
}

\begin{abstract}
Structural health monitoring is a key factor in life cycle management of infrastructures. Truly distributed fiber optic sensors are able to provide relevant information on large structures, such as nuclear power plants or nuclear waste disposal facilities. The sensing chain includes an optoelectronic unit and a sensing cable made of one or more optical fibers. A new instrument based on Optical Frequency Domain Reflectometry (OFDR), enables to perform temperature and strain measurements with a centimeter scale spatial resolution over hundred of meters and with a level of precision equal to $1 \mu$ strain and $0.1^{\circ} \mathrm{C}$. Several sensing cables are designed with different materials targeting to last for decades, either embedded in the concrete or attached to the surface of the structure. They must ensure an optimal transfer of temperature and strain from the concrete matrix to the optical fiber. Based on the European guide FD CEN/TR 14748 "Non-destructive testing - Methodology for qualification of non-destructive tests", a qualification method was developed. Tests were carried out using various sensing cables embedded in the volume or fixed to the surface of plain concrete specimens and representative-scale reinforced concrete structural elements. Measurements were performed with an OFDR instrument, while mechanical solicitations were imposed to the concrete element. Preliminary experiments seem very promising since measurements performed with distributed sensing systems are found comparable to values obtained with conventional sensors used in civil engineering and with the Strength of Materials Modelling. Moreover, the distributed sensing system makes it possible to detect and localize cracks appearing in concrete during the mechanical loading.
\end{abstract}

\section{INTRODUCTION}

Durability of structures is a crucial issue in civil engineering, involving major economical and environmental repercussions. Fundamental issues such as optimization of the maintenance strategy and extension of the service life with regard to safety have been among practical concerns facing infrastructure owners [1]. Structural Health Monitoring (SHM) is a promising approach which allows a real-time diagnosis of the state of wear or damage of an infrastructure. To be efficient, SHM techniques must provide relevant information on the integrity or functionalities of large civil structures like bridges, dams, dykes, nuclear power plants including containment buildings and cooling towers, deep or surface nuclear disposal facilities, etc. One of the major difficulties is the representativeness of measurements performed by sensors. Distributed Optical Fiber Sensing (DOFS) systems are an attractive tool for SHM [2]. Optical fibers consist in light waveguides made of silica glass, and are commonly used in the telecommunication industry. They are light, small (outer diameter around $150 \mu \mathrm{m}$ ) and insensitive to electromagnetic fields. Thanks to the low attenuation of the transmitted light signal, they enable

ae-mail: jean-marie.henault@edf.fr

This is an Open Access article distributed under the terms of the Creative Commons Attribution-Noncommercial License 3.0, which permits unrestricted use, distribution, and reproduction in any noncommercial medium, provided the original work is properly cited. 


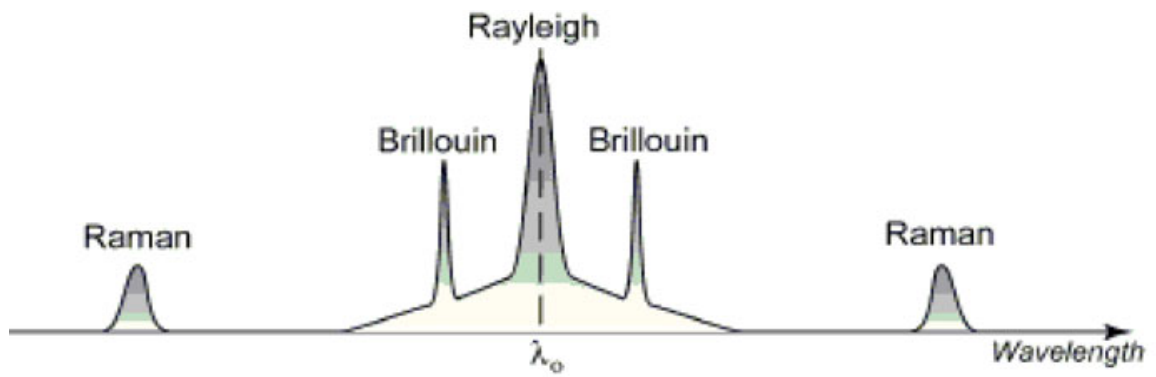

Figure 1. Scheme of silica scattering spectrum.

to perform long range transmissions, in the kilometre range. In DOFS, optical fibers act not only as the transmission medium but also as a continuous transducer. A DOFS system can be viewed as a few thousands of multiplexed measurement points obtained using an interrogation unit paired with an optical fiber. The only limitations are the spatial resolution of the instrument (inversely proportional to the maximal distance range) and its sensitivity. DOFS should be considered as a sensing solution complementary to short or long-gauge sensors commonly used in civil engineering.

\section{DOFS BASED ON RAYLEIGH BACKSCATTERING ANALYSIS BY OPTICAL FREQUENCY DOMAIN REFLECTOMETRY}

DOFS system is composed of an interrogation unit paired with an optical fiber. It enables to monitor the quantity subject to measurement, ie the measurand, continuously along the sensing fiber. In other words, the collected signal corresponds to the continuous record of the measurand under consideration as a function of the position along the fiber [3]. The working principle of DOFS is based on the analysis of the backscattered light in silica. When a light pulse propagates along an optical fiber, silica interacts continuously in space by scattering [4]. As presented in Figure 1, the spectrum of the scattered light shows elastic and inelastic components. There are three important types of scattering in optical fibers that can be exploited in distributed sensing. They are Rayleigh, Raman and Brillouin scattering. Rayleigh scattering is due to fluctuations of silica density and composition, Raman effect, to thermal molecular vibration, and Brillouin scattering to photons - phonons interactions. Analysis of the backscattered light may provide information on two measurands, temperature and strain, since Raman effect is temperature sensitive and Brillouin and Rayleigh effects are both temperature and strain sensitive. Measurand profiles along the fiber can be obtained by two main interrogation methods: Optical Time Domain Reflectometry (OTDR) and Optical Frequency Domain Reflectometry (OFDR). In OTDR, a laser pulse is used and the location is calculated considering the time of flight measurement. In OFDR, a swept frequency pulse is used to interact with the optical fiber. This last method is considered in our experiments.

Fundamentals and operating principles of Rayleigh OFDR systems are explained in great details in [5] and [6]. The system operates by first measuring and storing the Rayleigh scatter signature of the fiber at an initial state. Then, the scatter profile is measured at a later time with a strain or a temperature perturbation applied to the fiber. Data sets are broken into intervals along the fiber and Fourier transformed into the optical frequency domain. To determine the spectral shift between the reference and perturbed scans, a cross correlation is performed for each fiber interval. Any change in strain or temperature results in a shift in the correlation peak. A distributed measurement is formed by compiling the spectral shifts for each interval along the fiber. The relationship between the spectral shift and the change in strain and temperature is given by Equation (1).

$$
\Delta v_{F O}=C_{\varepsilon_{F O}} \varepsilon_{F O}+C_{T_{F O}} \Delta T_{F O}
$$


where $\Delta v_{F O}$ is the spectral shift, $\varepsilon_{F O}$ the fiber strain, $\Delta T_{F O}$ the fiber temperature change, $C_{\varepsilon F O}$ and $C_{T F O}$ are calibration constants. For standard single-mode fiber of type G652, at $1550 \mathrm{~nm}$, typical values of the latter constants are respectively $-0.1499 \mathrm{GHz} / \varepsilon$ and $-1.248 \mathrm{GHz} /{ }^{\circ} \mathrm{C}[6]$.

Currently, the only Rayleigh OFDR interrogation unit available on the market is the Optical Backscatter Reflectometer (OBR) from Luna Technologies, with a centimeter scale spatial resolution over hundred of meters and with a level of precision equal to $1 \mu$ strain and $0.1{ }^{\circ} \mathrm{C}$.

As previously mentioned, the DOFS system is composed of an interrogation unit and an optical fiber. With regard to industrial applications, an optical fiber is too fragile to be used directly. For this reason, the optical fiber (or several fibers) has to be wrapped by an additional coating to form a cable with an outer diameter of few $\mathrm{mm}$. The aim of this coating is to protect the optical fiber against mechanical and chemical aggressions and environmental conditions. For a sensing purpose, the coating must also be designed to ensure an optimal transfer of temperature and strain from the concrete matrix to the optical fiber. Several sensing cables have been identified for their potential interest to serve as sensors. Some of them could be used embedded in concrete and other ones could be mounted on the surface of the structure. Up to now, these cables have not been exhaustively tested by the suppliers and their lifetime has still to be evaluated for cementitious composites applications. As a consequence, further work should be undertaken to study and improve the capabilities of these sensing cables for measuring temperature and strain over time.

\section{PERFORMANCES OF SENSING CABLES FOR THE MONITORING OF CONCRETE STRUCTURES}

Based on the European guide for qualification of non-destructive tests 7, evaluation of the sensing cable performances is conducted through a "from the lab to the field" methodology [8]. Two main issues have to be considered. First, for strain measurement, a substantial level of adherence is required between the optical fiber and the surrounding coating, as well as between the coating and concrete in order to get an optimal transfer of strain from the concrete matrix to the fiber [9]. Secondly, a good durability of the sensing cable is mandatory as the cable is expected to last for decades especially when embedded in concrete and without any possibility of replacement. To date, chemical inertness of the external coating in alkaline environments such as in concrete is not clearly established.

Evaluation is conducted step-by-step to assess the influence of different parameters on the performances of a given sensing cable, as illustrated on Figure 2. Thermal and mechanical loadings are applied to the cable subjected to various environments: (i) in controlled benches out of concrete, (ii) in mortar and concrete samples, (iii) in reinforced concrete (RC) beam, and finally (iv) in real structures. In parallel, ageing tests have to be carried out. At each stage, distributed measurements are performed with an OBR device and results are compared to those obtained from conventional sensors used in civil engineering and from a Strength of Materials Modelling (SMM) approach.

This evaluation program began at the end of 2009 and will continue over the next 3 years. In the following paragraphs, preliminary results are presented and discussed.

\subsection{Concrete specimens tested under compression loading}

In this experimental campaign, a wave-like composite sensing cable, made of fiber reinforced epoxy resin, with an average diameter of $5 \mathrm{~mm}$ [10] (Figure 3(a)), was embedded into the centre of a concrete cylinder of height $100 \mathrm{~cm}$ and diameter $16 \mathrm{~cm}$. Distributed measurements were performed using an OBR device. The cylinder was also equipped with three $50 \mathrm{~cm}$ invar bars fixed to the concrete surface and equipped with a Linear Variable Differential Transducer (LVDT). These specimens were then tested under compression loading, as illustrated in Figure 3(b). Strain variation obtained with the two sensing systems after completion of the compressive loading is presented in Figure 3(c). The distributed sensing system provides multi-points measurements over the $50 \mathrm{~cm}$ gauge length of the LVDT system. The 


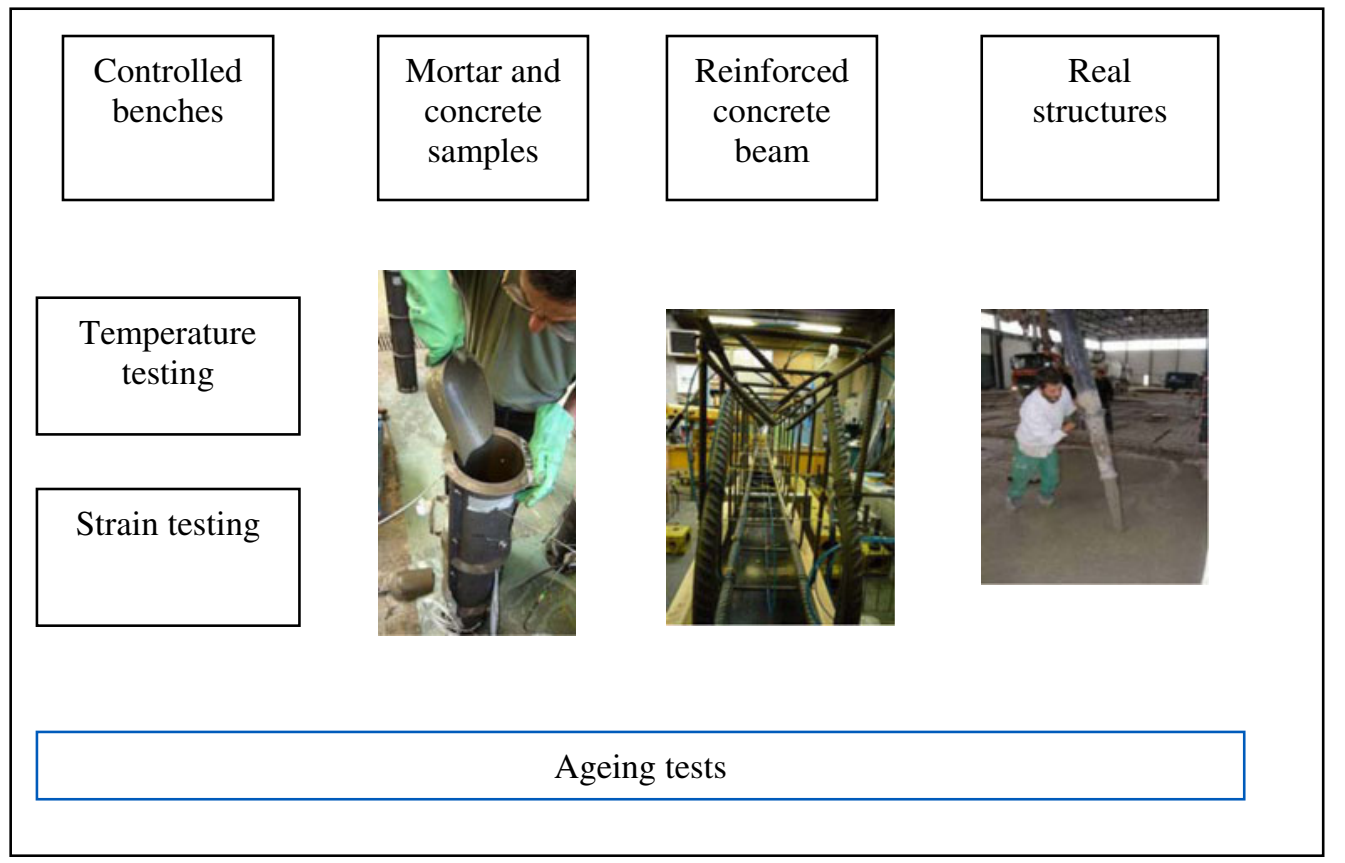

Figure 2. Evaluation methodology.
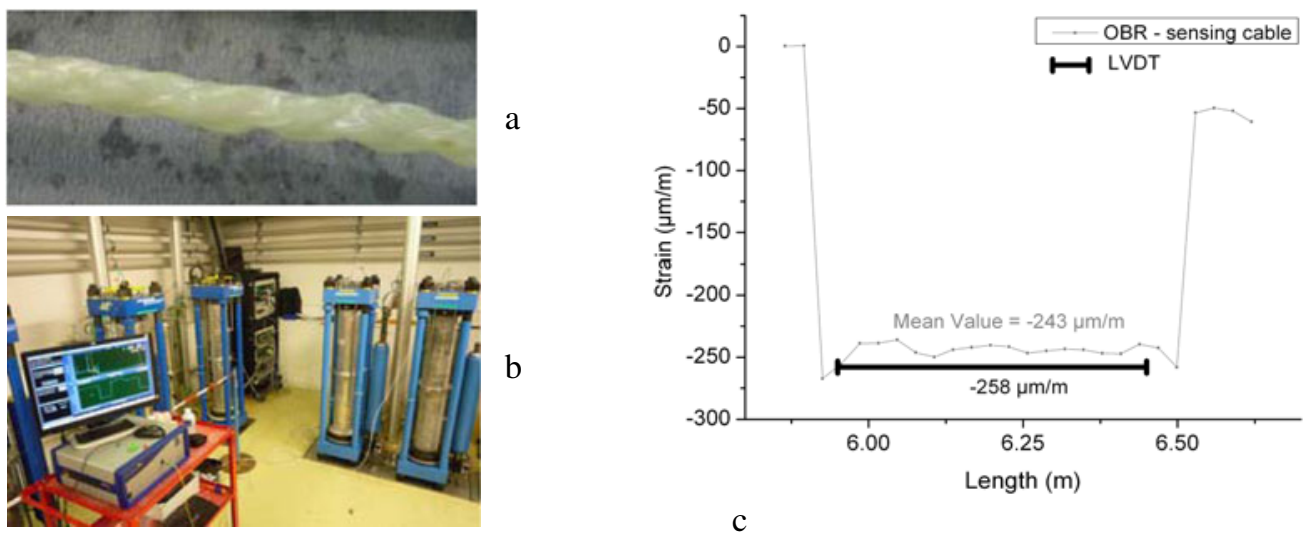

Figure 3. Pictures of the wave-like composite cable (a) and the testing bench (b) - Compared results of the distributed and conventional sensing systems (c).

values are roughly constant which is in accordance with SMM. Small fluctuations may result from the heterogeneous composition of concrete (mortar and aggregates) which is not taken into account in SMM, or from an imperfect bonding at the concrete/cable coating interface. In order to provide a relevant comparison with LVDT measurements, values are averaged over the same span. The average value of the distributed sensing system is found to be comparable to that obtained with conventional LVDT sensor. The observed deviation (less than 6\%) may be explained either by the different locations of the sensors, as the sensing cable is embedded in the core of the concrete specimen while the LVDT sensor is attached to the concrete surface, or by an imperfect bonding between the sensing cable and concrete. 
AMP 2010
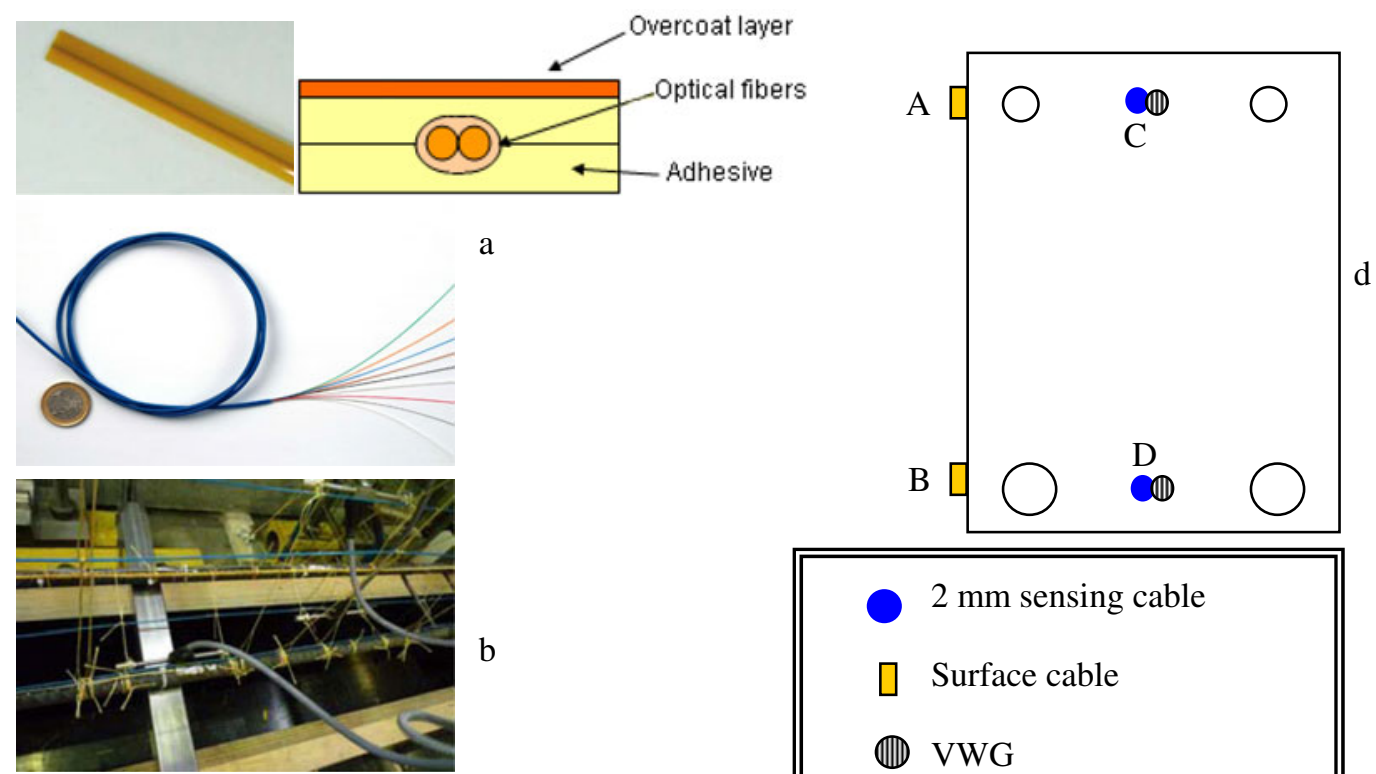

a

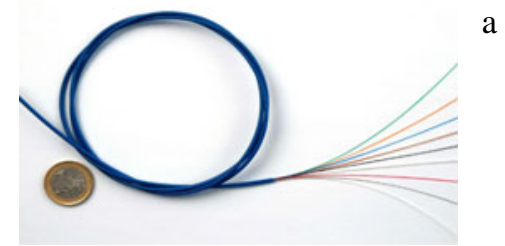

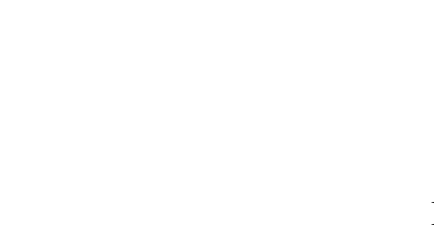

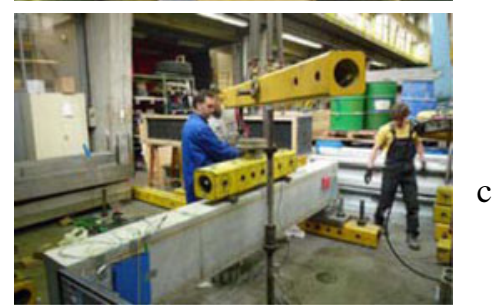

$2 \mathrm{~mm}$ sensing cable

$\mathrm{b}$

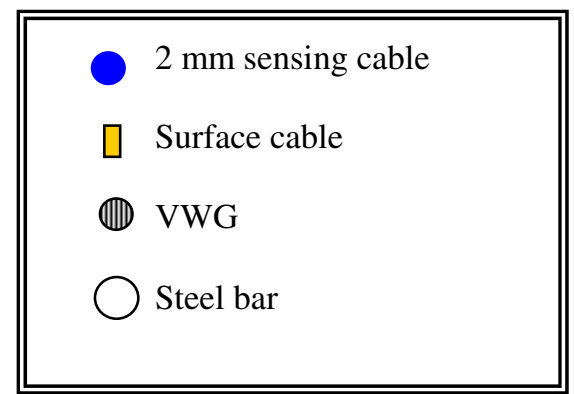

Figure 4. Pictures of the sensing cables (a), the installation of the sensors in the beam (b) and the 4 point testing facility (c) - scheme of the instrumentation (d).

\subsection{Reinforced concrete beam under 4 points bending test}

In this experiment, as illustrated in Figure 4, a $340 \times 50 \times 25 \mathrm{~cm} 3 \mathrm{RC}$ beam was equipped with several sensing systems. A multifiber cable with a polymer jacket (diameter $2 \mathrm{~mm}$ ) and Vibrating Wire Gauges (VWG) were embedded in the central part of the beam. After pouring and hardening of concrete, a specific sensing cable, in which the optical fiber is included between two polymer adhesive layers, was bonded on the surface of the beam, at the same depth in front of the embedded sensors. The beam was loaded step by step under a 4 point bending test, with a distance of $85 \mathrm{~cm}$ between the two central points. Results obtained at load levels of $20 \mathrm{kN}$ and $100 \mathrm{kN}$ are presented in Figure 5. In both cases, distributed measurements give comparable results with regard to VWG. Moreover, at a load level of $20 \mathrm{kN}$, strains remain low and concrete exhibits an elastic behaviour in the whole beam. Distributed sensing profiles in tension and compression both show a trapezoid shape between the 4 points of the testing bench as expected from SMM. For the load level of $100 \mathrm{kN}$, concrete tensile stress exceeds the ultimate strength and cracks appear in the lower part of the beam which is under tension. Strain peaks are observed on the corresponding distributed sensing profiles at locations compatible with the crack locations identified by visual inspection. Therefore, the measuring system might enable to detect and localize cracks in concrete. The specific shape of the strain peaks seems to be related to the mechanical response of the cable while transferring the crack opening from the concrete to the optical fiber by shear stress within the coating material. Such a mechanical response is specific to each cable, depending on the geometry and the mechanical properties of the constitutive materials of the cable [11], which is confirmed in Figure 5 

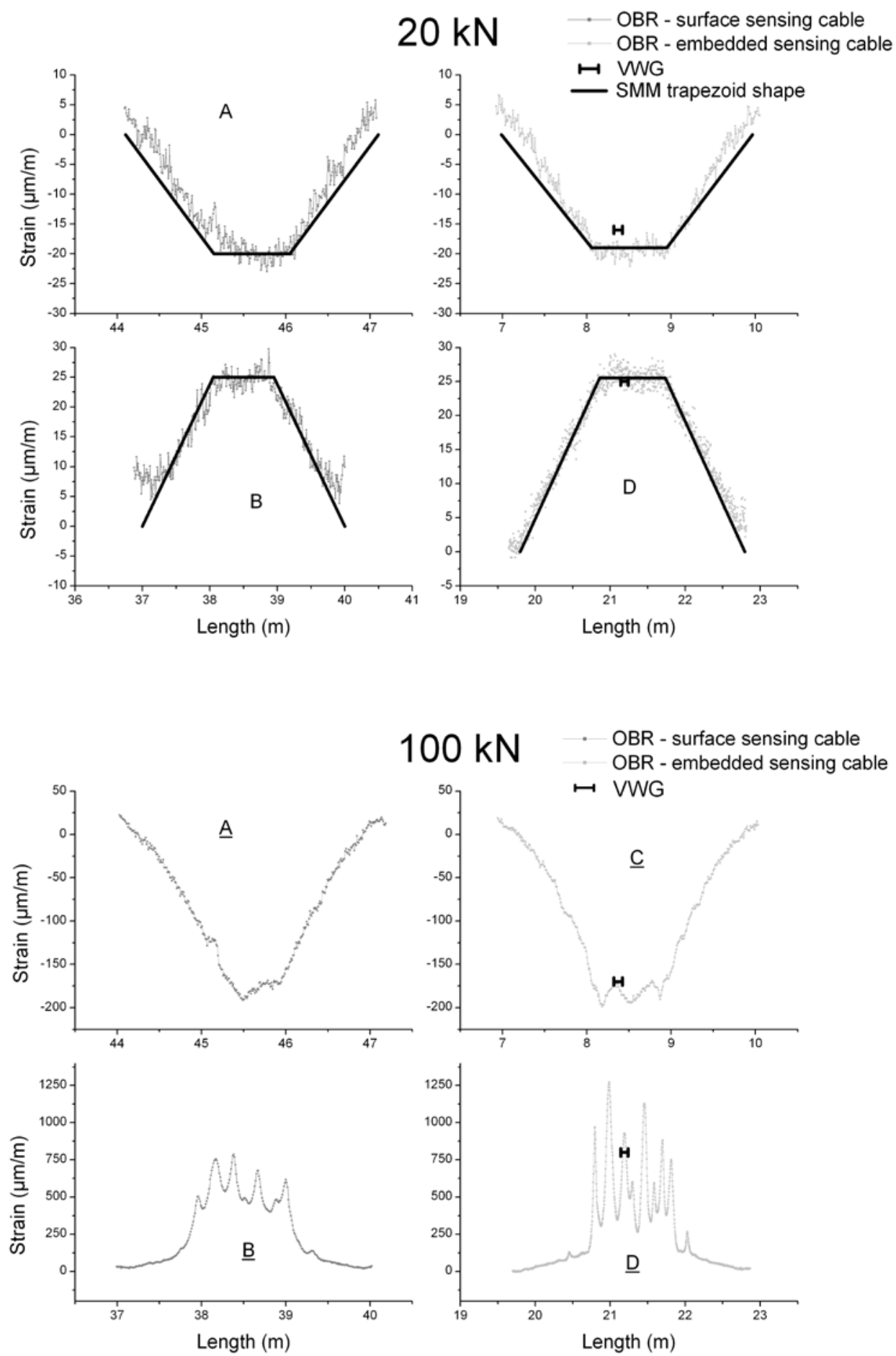

Figure 5. Compared results of the sensing systems obtained at load levels of $20 \mathrm{kN}$ and $100 \mathrm{kN}$, for the sensors in the upper part of the beam under compression (surface A and embedded C) and in the lower part of the beam subjected to tension(surface B and embedded D). 


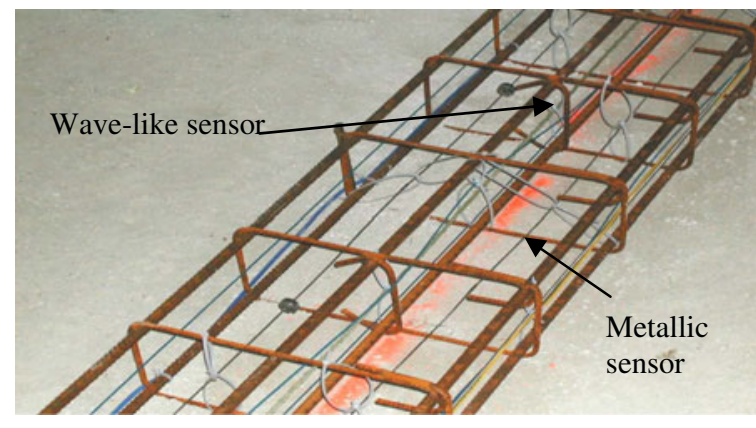

a

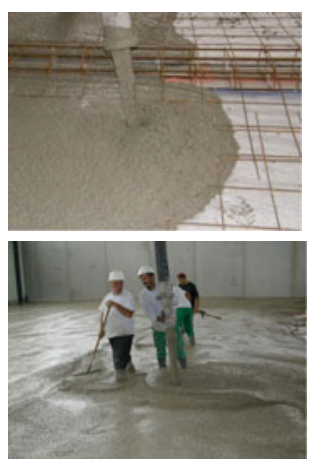

b

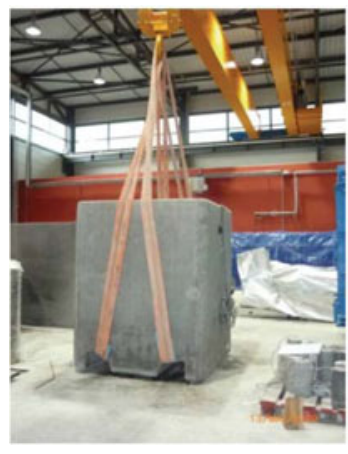

c

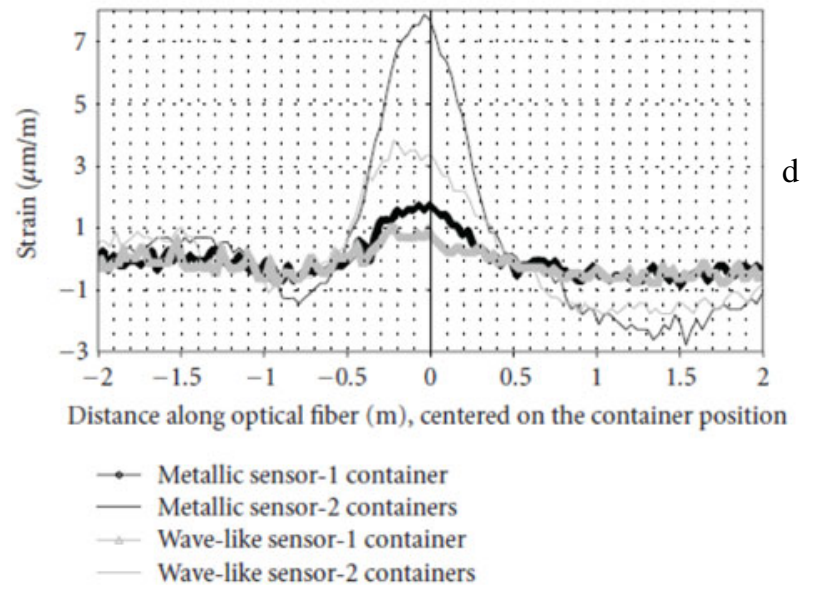

Figure 6. Pictures of the slab during instrumentation (a), pouring and working activities (b) and container test (c) - Strain profiles provided by the distributed sensing system with 2 different cables (d).

( $100 \mathrm{kN}, \mathrm{B}$ and D) by the different shapes of the strain peaks provided by embedded or surface mounted cables. Further experimental work and modelling are needed to evaluate the mechanical response of each cable, in order to assess quantitatively the crack opening phenomenon by distributed measurements.

\subsection{Field experiments}

In this experiment, several types of cables were embedded in a concrete building slab, as detailed in reference [8]. The first goal of this test was to assess the robustness of the cable under harsh conditions similar to that occurring on working sites, and especially during the concrete pouring stage, as shown in Figure 6. No fiber breaking was observed during the experiment. A few months after pouring, a qualitative test was performed by lifting and piling-up heavy containers of about 12 tons with an overhead travelling crane above the instrumented zone. All the fibers were connected to form a loop and were paired with the OBR device. The mechanical load due to the container was detected and localized with all the cables. Slight discrepancies were observed between the various sensors, which might be explained by the different locations of the cables within the slab and by differences in the cable sensing performances. 


\section{CONCLUSION AND PERSPECTIVES}

Distributed Optical Fiber Sensing systems fit Structural Health Monitoring needs, as they can provide relevant information over large structures with only limited equipment. One interrogation unit paired with one fiber can provide few thousands of multiplexed measurement points. Interrogation method based on Optical Frequency Domain Reflectometry and analysis of the Rayleigh backscattering provide very high spatial resolution (centimetre-scale) and high sensitivity to temperature and strain. Optical fibers cannot be used directly with their primary coating They must be wrapped by a few mm thick coating to form a cable, so as to be protected against mechanical and chemical aggressions. This coating must also be designed to ensure an optimal transfer of temperature and strain from the concrete to the optical fiber, but performances and durability of the sensing cables have to be rigorously evaluated. Preliminary results of a large "from the lab to the field" evaluation are presented in this paper, with embedded and surface mounted cables. These first experiments are very promising since measurements performed with distributed sensing system are comparable to values obtained with conventional sensors used in civil engineering and with the Strength of Materials Modelling. Moreover, the distributed sensing system makes it possible to detect and localize cracks appearing in concrete during the mechanical loading. In addition, field experiments have demonstrated that cables were resistant to concrete pouring and working activities. In a near future, these preliminary tests will be completed by laboratory temperature testing, bond strength assessment at the various interfaces (fiber / coating / concrete) and crack opening quantification. Degradation and long-term stability of the sensing cables submitted to the severe conditions associated with the construction environments of civil infrastructure is another crucial issue which needs to be investigated.

\section{References}

[1] Guidance for Optimizing Nuclear Power Plant Maintenance Programmes, IAEA TECDOC Series No. 1383

[2] B. Glisic and D. Inaudi, Fibre opic methods for structural health monitoring (Wiley, 2007)

[3] A. Hartog, Distributed Fiber-Optic Sensors : Principles and applications, 251-301 (Optical Fiber sensor Technology, 2000)

[4] G. Agrawal, Non-linear fiber optics (Academic Press, 2001)

[5] B. Soller, D Gifford, M. Wolfe and M. Froggatt, Optics Express, 13, 666-674 (2005)

[6] A. Sang, M. Froggatt, D. Gifford, S. Kreger and B. Dickerson, IEEE Sensors Journal, 8, 13751380 (2008)

[7] European guide FD CEN/TR 14748, Non-destructive testing. Methodology for qualification of non-destructive tests (2005)

[8] J.M. Henault, G. Moreau, S. Blairon, J. Salin, J.R. Courivaud, F. Taillade, E. Merliot, J.P. Dubois, J. Bertrand, S. Buschaert, S. Mayer and S. Delepine-Lesoille, Advances in Civil Engineering, 2010, Article ID 930796 (2010)

[9] F. Ansari and Y. Libo, Journal of Engineering Mechanics, 124, 385-394 (1998)

[10] S. Delepine-Lesoille, E. Merliot, C. Boulay, L. Quétel, M. Delaveau, and A. Courteville, Smart Mater. Struct., 15, 931-38 (2006)

[11] G. Duck, G. Renaud and R. Measures, Smart Mater. Struct., 8, 175-181 (1999) 